\title{
On the correspondence between quantum and classical variational principles
}

\author{
D. E. Ruiz and I. Y. Dodin \\ Department of Astrophysical Sciences, Princeton University, Princeton, New Jersey 08544, USA
}

\begin{abstract}
Classical variational principles can be deduced from quantum variational principles via formal reparameterization of the latter. It is shown that such reparameterization is possible without invoking any assumptions other than classicality and without appealing to dynamical equations. As examples, first principle variational formulations of classical point-particle and cold-fluid motion are derived from their quantum counterparts for Schrödinger, Pauli, and Klein-Gordon particles.
\end{abstract}

\section{INTRODUCTION}

It is commonly known that variational methods are a powerful tool for studying the dynamics of various physical systems, e.g., quantum molecular dynamics [1, 2], fluid mechanics [3, 4], classical motion of single particles and collective processes in plasmas [5, 6], and wave propagation in both linear and nonlinear media [7-10]. Variational formulations are advantageous as they lead to dynamical equations in a manifestly conservative form derived from a single scalar function, a Lagrangian (or Lagrangian density). The fact that these equations can be approximated robustly and self-consistently by approximating just one function makes the method particularly attractive for reduced calculations [5, 11, 12]. However, so far, exact Lagrangians have been obtained largely heuristically or ad hoc [13]. Such approaches tend to obscure the physical meaning of the results and limit their applicability, while regular ways to deduce Lagrangians rigorously from first principles are yet to be found.

Here we show that classical variational principles (VPs) can be deduced from quantum VPs, which are well known, via formal reparameterization of the latter. Such reparameterization is possible without appealing to dynamical equations and without invoking any assumptions other than classicality. This distinguishes our theory from the existing variational formulations of the quantum-classical correspondence, which are more restrictive [14]. Also as a complement to those formulations, we consider both single-particle and fluid VPs and rigorously explain how they are connected through quantum VPs. Classical-fluid Lagrangians flow as the semiclassical limit of the fundamental quantum Lagrangian (FQL), Eq. (3), and the point-particle Lagrangians are then yielded as corollaries for narrow but otherwise arbitrary wave packets. This approach enables the first principle classical Lagrangian description of most general, relativistic vector particles (e.g., a Dirac electron) and, similarly, the geometrical optics (GO) description of any vector waves, as we discuss in a companion paper [15]. The present paper is intended as an introduction to such calculations. Thus, below, we primarily focus on systematization of VPs for commonly known systems, including Schrödinger, Pauli, and Klein-Gordon particles.

We explicitly show how to deduce classical Lagrangians for these particles and the corresponding fluids from the FQL. In particular, we show that the expression for the Bohm quantum potential [16, 17], the so-called Weizsäcker correction [18], the Madelung equations [19], and the Chen-Sudan Lagrangian density [20] all emerge naturally within our unifying theory as special cases. We also obtain an alternative, manifestly Lagrangian representation of Takabayasi equations for a Pauli particle. In contrast to the original theory [21], our model yields the dynamics of the spin vector $\mathbf{S}$ directly from the VP and employs two, rather than three, equations; hence, $S=1 / 2$ is ensured irrespective of initial conditions.

The paper is organized as follows. In Sec. II, we define the basic notation. In Sec. III, we introduce the general formulas. In Sec. IV, we discuss a Schrödinger particle and the Madelung equations. In Sec. V, we discuss a Pauli particle and the variational formulation of Takabayasi equations. In Sec. VI, we discuss a Klein-Gordon particle and rederive the Chen-Sudan fluid Lagrangian density. In Sec. VII, we summarize our main results and outline future applications of the proposed theory.

\section{NOTATION}

The following notation is used throughout the paper. The symbol "戸" denotes definitions. We use natural units, so the speed of light equals one $(c=1)$, and so is the Planck constant $(\hbar=1)$. The Minkowski metric is adopted with signature $(-,+,+,+)$, so, in particular, $\mathrm{d}^{4} x \equiv \mathrm{d} t \mathrm{~d}^{3} x$. Generalizations to curved metrics are straightforward to apply [22]. Greek indexes span from 0 to 3 and refer to spacetime coordinates, $x^{\mu}$, with $x^{0}$ corresponding to the time variable, $t$; in particular, $\partial_{\mu} \equiv \partial / \partial x^{\mu}$. Latin indexes span from 1 to 3 and denote the spatial variables, $x^{i}$ (except where specified otherwise); in particular, $\partial_{i} \equiv \partial / \partial x^{i}$. Summation over repeated indexes is assumed. Also, in the Euler-Lagrange equations (ELEs), the notation " $\delta a$ :" denotes, as usual, that the corresponding equation was obtained by extremizing the action integral with respect to $a$. 


\section{BASIC EQUATIONS}

\section{A. Fundamental quantum Lagrangian}

The dynamics of a quantum particle is governed by the least action principle,

$$
\delta \Lambda=0
$$

where $\Lambda$ is the action integral,

$$
\Lambda=\int \mathfrak{L} \mathrm{d}^{4} x
$$

and $\mathfrak{L}$ is the Lagrangian density. Like for any other nondissipative linear wave [22], the Lagrangian density of a quantum particle can be expressed as [23],

$$
\mathfrak{L}=\frac{i}{2}\left[\psi^{\dagger}\left(\partial_{t} \psi\right)-\left(\partial_{t} \psi^{\dagger}\right) \psi\right]-\psi^{\dagger} \hat{H} \psi
$$

which is what we term (the density of) the FQL. Here $\hat{H}$ is some Hermitian operator called Hamiltonian, $\psi$ is a complex vector field ("state function"), and $\psi^{\dagger}$ is its adjoint. Treating $\psi$ and $\psi^{\dagger}$ as independent functions [22], one obtains from Eq. (1) two mutually adjoint ELEs,

$$
\begin{aligned}
\delta \psi^{\dagger}: & i \partial_{t} \psi=\hat{H} \psi, \\
\delta \psi: & -i \partial_{t} \psi^{\dagger}=(\hat{H} \psi)^{\dagger} .
\end{aligned}
$$

It is also possible to derive other, different equations from Eqs. (3) if $\mathfrak{L}$ is reparameterized, i.e., if other independent functions are chosen instead of $\psi$ and $\psi^{\dagger}$. This can be done in general, using the fact that the Hamiltonian always can be expressed as $\hat{H}=H(t, \hat{\mathbf{x}}, \hat{\mathbf{p}})[22]$ (here $H$ is some matrix function, $\hat{\mathbf{x}}=\mathbf{x}$ is the position operator, $\hat{\mathbf{p}}=-i \boldsymbol{\nabla}$ is the momentum operator, and the standard coordinate representation is assumed), so

$$
\mathfrak{L}=\frac{i}{2}\left[\psi^{\dagger}\left(\partial_{t} \psi\right)-\left(\partial_{t} \psi^{\dagger}\right) \psi\right]-\psi^{\dagger} H(t, \mathbf{x},-i \nabla) \psi
$$

Specifically, the classical reparameterization of this Lagrangian density is performed as follows.

\section{B. Single-particle Lagrangian}

First, let us consider the simplest classical reparameterization of Eq. (6). For a scalar particle, the state function can be expressed as

$$
\psi=a e^{i \theta}
$$

where the amplitude $a \equiv \sqrt{\mathcal{I}}$ and the phase $\theta$ are real scalar functions of $(t, \mathbf{x})$. Let us assume the semiclassical limit, i.e., that $\mathcal{I}(t, \mathbf{x})$ is slow compared to $\theta(t, \mathbf{x})$. Then, Eq. (6) gives [24]

$$
\mathfrak{L} \approx-\mathcal{I}\left[\partial_{t} \theta+H(t, \mathbf{x}, \boldsymbol{\nabla} \theta)\right]
$$

which one may recognize as Hayes's representation of the Lagrangian density of a GO wave [25]. Treating $\theta$ and $\mathcal{I}$ as independent functions, one hence arrives at the following ELEs:

$$
\begin{array}{ll}
\delta \theta: & \partial_{t} \mathcal{I}+\nabla \cdot(\mathcal{I} \mathbf{v})=0 \\
\delta \mathcal{I}: & \partial_{t} \theta+H(t, \mathbf{x}, \boldsymbol{\nabla} \theta)=0
\end{array}
$$

where $\mathbf{v} \doteq \partial_{\mathbf{p}} H(t, \mathbf{x}, \mathbf{p})$ is the group velocity evaluated at the wave vector $\mathbf{p}(t, \mathbf{x})=\boldsymbol{\nabla} \theta$. Equation (9) is a continuity equation known as the action conservation theorem (ACT). Equation (10) is the Hamilton-Jacobi representation of the local dispersion relation,

$$
\mathcal{E}=H(t, \mathbf{x}, \mathbf{p})
$$

where $\mathcal{E} \doteq-\partial_{t} \theta$ is the local frequency.

To the extent that a wave packet is well localized such that it is meaningful to describe its dynamics as the dynamics of the packet's geometrical center, the continuouswave description can be replaced with a simpler, pointparticle model. In this case, one can approximate the action density with a delta function,

$$
\mathcal{I}(t, \mathbf{x})=\delta(\mathbf{x}-\mathbf{X}(t))
$$

so the LD can be replaced with just a point-particle Lagrangian, $L \doteq \int \mathfrak{L} \mathrm{d}^{3} x$. This is done as follows. Let us express the complete action integral, $\Lambda$, in the form

$$
\begin{aligned}
\Lambda=\underbrace{-\int \mathcal{I}(t, \mathbf{x}) \partial_{t} \theta(t, \mathbf{x}) \mathrm{d}^{4} x}_{\Lambda_{1}} \\
\underbrace{-\int \mathcal{I}(t, \mathbf{x}) H(t, \mathbf{x}, \nabla \theta(t, \mathbf{x})) \mathrm{d}^{4} x}_{\Lambda_{2}} .
\end{aligned}
$$

Then, substituting Eq. (12) leads to

$$
\begin{aligned}
\Lambda_{1} & =-\int \delta(\mathbf{x}-\mathbf{X}(t)) \partial_{t} \theta(t, \mathbf{x}) \mathrm{d}^{4} x \\
& =\int\left[\partial_{t} \delta(\mathbf{x}-\mathbf{X}(t))\right] \theta(t, \mathbf{x}) \mathrm{d}^{4} x \\
& =\int[-\nabla \delta(\mathbf{x}-\mathbf{X}(t))] \cdot \dot{\mathbf{X}}(t) \theta(t, \mathbf{x}) \mathrm{d}^{4} x \\
& =\int\left[-\int \theta(t, \mathbf{x}) \boldsymbol{\nabla} \delta(\mathbf{x}-\mathbf{X}(t)) \mathrm{d}^{3} x\right] \cdot \dot{\mathbf{X}}(t) \mathrm{d} t \\
& =\int\left[\int \delta(\mathbf{x}-\mathbf{X}(t)) \nabla \theta(t, \mathbf{x}) \mathrm{d}^{3} x\right] \cdot \dot{\mathbf{X}}(t) \mathrm{d} t \\
& =\int \mathbf{P}(t) \cdot \dot{\mathbf{X}}(t) \mathrm{d} t,
\end{aligned}
$$

where $\mathbf{P}(t) \doteq \nabla \theta(t, \mathbf{X}(t))$. Similarly,

$$
\Lambda_{2}=-\int H(t, \mathbf{X}(t), \mathbf{P}(t)) \mathrm{d} t .
$$


Thus, the total action integral is expressed as $\Lambda=\int L \mathrm{~d} t$. The function $L$ serves as a point-particle Lagrangian and is given by

$$
L=\mathbf{P} \cdot \dot{\mathbf{X}}-H(t, \mathbf{X}, \mathbf{P}) .
$$

One may recognize this $L$ as the standard classical Lagrangian, where $\mathbf{X}$ and $\mathbf{P}$ serve as a canonical coordinate and a canonical momentum, respectively. Treating them as independent variables then leads [26, Sec. 43] to ELEs matching Hamilton's equations in the canonical form,

$$
\begin{array}{ll}
\delta \mathbf{P}: & \dot{\mathbf{X}}=\partial_{\mathbf{P}} H(t, \mathbf{X}, \mathbf{P}), \\
\delta \mathbf{X}: & \dot{\mathbf{P}}=-\partial_{\mathbf{X}} H(t, \mathbf{X}, \mathbf{P}) .
\end{array}
$$

(Hence, $\mathcal{E}$ and $\mathbf{p}$ can be understood as local canonical energy and momentum.) Equations (17) and (18) also serve as ray equations for Eqs. (9) and (10) [22].

It is to be noted that our derivation does not require interpreting $\psi$ as a probability and holds for any $H$. It is also to be noted that our point-particle equations are derived without assuming a specific profile of $\mathcal{I}(t, \mathbf{x})$. All we require instead is that this function be narrow enough (yet wide enough compared to the particle wavelength; otherwise, the GO approximation does not apply).

\section{Fluid Lagrangian density}

Now consider an ensemble of particles described by $\left(\theta_{q}, \mathcal{I}_{q}\right), q=1, \ldots N$. Assuming the energy of particle interactions (except through average fields included in $H$ ) is negligible, the ensemble Lagrangian density $\mathfrak{L}$ equals the sum of single-particle Lagrangians $\mathfrak{L}_{q}$ given by Eq. (8). Let us also assume that particles are cold. This means that all $\mathbf{p}_{q}$ are about the same, and thus so are all $\mathcal{E}_{q}$; i.e.,

$$
\nabla \theta_{q} \approx \nabla \theta, \quad \partial_{t} \theta_{q} \approx \partial_{t} \theta,
$$

where $\theta$ is some average phase. Then, the ensemble is described by the same $\mathfrak{L}$ as the one given by Eq. (8), except $\mathcal{I}$ must be replaced with the particle density,

$$
n \doteq \sum_{q=1}^{N} \mathcal{I}_{q} .
$$

In other words, the ensemble's Lagrangian density is given by

$$
\mathfrak{L}=-n\left[\partial_{t} \theta+H(t, \mathbf{x}, \nabla \theta)\right] .
$$

The corresponding ELEs are

$$
\begin{array}{ll}
\delta \theta: & \partial_{t} n+\boldsymbol{\nabla} \cdot(n \mathbf{v})=0, \\
\delta n: & \partial_{t} \theta+H(t, \mathbf{x}, \boldsymbol{\nabla} \theta)=0,
\end{array}
$$

and $\mathbf{v}$ serves as the flow velocity. These equations provide a complete description of the ensemble as a cold fluid, so
$\mathfrak{L}$ given by Eq. (21) can be viewed as the Lagrangian density of a cold classical fluid. (Specific examples will be discussed in the next sections.) It is to be noted, like we already did before, that interpreting $\psi$ as a probability amplitude is not needed within this approach.

Thermal corrections for isentropic fluid also can be introduced readily, at least ad hoc. This is done by adding to $\mathfrak{L}$ a term $-n U(t, \mathbf{x}, n)$, which leads to

$$
\mathfrak{L}=-n\left[\partial_{t} \theta+H(t, \mathbf{x}, \boldsymbol{\nabla} \theta)+U(t, \mathbf{x}, n)\right] .
$$

From comparing Eqs. (21) and (24), it is seen that $U$ has the meaning of the per-particle internal energy; then $n^{2} \partial_{n} U$ serves as the pressure [27].

Outside the ad hoc approach, there exists other variational methods to obtain corrections to the cold-fluid variational principle that we derived. In Ref. [28], the Thomas-Fermi energy functional is minimized in order to obtain the quantum hydrodynamic equations with thermal gradient corrections included. Also, the Lagrangian density for more general, non-isentropic fluids [3] can be deduced from the FQL. Details will be reported in a separate paper. Below, we will completely ignore thermal effects for clarity.

\section{SCHRÖDINGER PARTICLE}

Let us specifically discuss a Schrödinger particle, i.e., a nonrelativistic charged particle governed by

$$
\hat{H}=\frac{1}{2 m}(-i \boldsymbol{\nabla}-q \mathbf{A})^{2}+q \varphi .
$$

Here $m$ and $q$ are the particle mass and charge, $\mathbf{A}=$ $\mathbf{A}(t, \mathbf{x})$ is the vector potential, and $\varphi=\varphi(t, \mathbf{x})$ is the scalar potential. Substitution of Eq. (7) leads to the following exact Lagrangian density,

$$
\begin{gathered}
\mathfrak{L}=-\mathcal{I}\left[\partial_{t} \theta+\frac{1}{2 m}(\boldsymbol{\nabla} \theta-q \mathbf{A})^{2}+q \varphi\right]-W_{\mathrm{S}} \\
W_{\mathrm{S}} \doteq \frac{(\boldsymbol{\nabla} a)^{2}}{2 m}=\frac{(\boldsymbol{\nabla} \mathcal{I})^{2}}{8 m \mathcal{I}}
\end{gathered}
$$

where $W_{\mathrm{S}}$ can be recognized as the Weizsäcker correction [18]. The corresponding ELEs are

$$
\begin{array}{ll}
\delta \theta: & \partial_{t} \mathcal{I}+\boldsymbol{\nabla} \cdot(\mathcal{I} \mathbf{v})=0, \\
\delta \mathcal{I}: & \partial_{t} \theta+\frac{1}{2 m}(\boldsymbol{\nabla} \theta-q \mathbf{A})^{2}+q \varphi+Q=0,
\end{array}
$$

where $\mathbf{v} \doteq(\nabla \theta-q \mathbf{A}) / m$, and

$$
Q \doteq-\frac{\nabla^{2} \sqrt{\mathcal{I}}}{2 m \sqrt{\mathcal{I}}}
$$

is recognized as the Bohm quantum potential [16, 17], which is obtained when varying the $\int W_{\mathrm{S}} \mathrm{d}^{4} x$ term of the action. Equation (28) represents the ACT, and Eq. (29) 
represents a quantum Hamilton-Jacobi equation. Taking the gradient of the latter readily yields

$$
m\left(\partial_{t}+\mathbf{v} \cdot \boldsymbol{\nabla}\right) \mathbf{v}=q(\mathbf{E}+\mathbf{v} \times \mathbf{B})-\nabla Q .
$$

Equations (28) and (31) combined together coincide with Madelung equations [19]. Hence, $\mathfrak{L}$ given by Eq. (26) can be identified as the Madelung Lagrangian density. The equation for cold classical fluid are obtained by replacing $\mathcal{I}$ with $n$, as in Sec. III C, and also by neglecting $Q$ in Eq. (31) or, equivalently, $W_{\mathrm{S}}$ in Eq. (26) (which constitutes the semiclassical, or GO approximation). Hence,

$$
\mathfrak{L}=-n\left[\partial_{t} \theta+\frac{1}{2 m}(\nabla \theta-q \mathbf{A})^{2}+q \varphi\right]
$$

can be identified as a Lagrangian density of a cold classical fluid, in agreement with Ref. [3]. According to Sec. III B, the classical point-particle motion is then governed by Eqs. (17) and (18) with the Hamiltonian

$$
H(t, \mathbf{X}, \mathbf{P})=\frac{1}{2 m}[\mathbf{P}-q \mathbf{A}(t, \mathbf{X})]^{2}+q \varphi(t, \mathbf{X}) .
$$

\section{PAULI PARTICLE}

Now let us consider a Pauli particle, i.e., a nonrelativistic spin-1/2 particle governed by [29]

$$
\hat{H}=\frac{1}{2 m}(-i \boldsymbol{\nabla}-q \mathbf{A})^{2}+q \varphi-\frac{q}{2 m} \boldsymbol{\sigma} \cdot \mathbf{B},
$$

where $\boldsymbol{\sigma}=\left(\sigma_{x}, \sigma_{y}, \sigma_{z}\right)$ are the Pauli matrices,

$$
\sigma_{x}=\left(\begin{array}{cc}
0 & 1 \\
1 & 0
\end{array}\right), \quad \sigma_{y}=\left(\begin{array}{cc}
0 & -i \\
i & 0
\end{array}\right), \quad \sigma_{z}=\left(\begin{array}{cc}
1 & 0 \\
0 & -1
\end{array}\right),
$$

and $\mathbf{B} \doteq \boldsymbol{\nabla} \times \mathbf{A}$ is magnetic field. [Replacing the Bohr magneton $q /(2 m)$ in Eq. (34) with a different constant would not significantly affect the below calculation.] The state function $\psi$ has two complex components; i.e.,

$$
\psi=\left(\begin{array}{l}
a_{1} e^{i \theta_{1}} \\
a_{2} e^{i \theta_{2}}
\end{array}\right)
$$

where $a_{1,2}$ and $\theta_{1,2}$ are real. Thus, it can be cast in the following general form, $\psi=e^{i \theta} \eta(\vartheta, \zeta) \sqrt{\mathcal{I}}$, with

$$
\eta(\vartheta, \zeta)=\left(\begin{array}{c}
e^{-i \vartheta / 2} \cos (\zeta / 2) \\
e^{i \vartheta / 2} \sin (\zeta / 2)
\end{array}\right)
$$

Here $\theta \doteq\left(\theta_{1}+\theta_{2}\right) / 2, \vartheta \doteq \theta_{2}-\theta_{1}, \mathcal{I} \doteq \psi^{\dagger} \psi, \zeta$ is real and defined via

$$
a_{1}=a \cos (\zeta / 2), \quad a_{2}=a \sin (\zeta / 2),
$$

and $a \doteq \sqrt{\mathcal{I}}$. Hence, the Lagrangian density can be expressed as

$$
\begin{aligned}
\mathfrak{L}=\frac{i \mathcal{I}}{2}\left[\eta^{\dagger}\left(d_{t} \eta\right)-\left(d_{t} \eta^{\dagger}\right) \eta\right]-\mathcal{I}\left(\partial_{t} \theta\right)-W_{\mathrm{P}} \\
-\mathcal{I}\left[\frac{1}{2 m}(\boldsymbol{\nabla} \theta-q \mathbf{A})^{2}+q \varphi+U_{\mathrm{SG}}\right]
\end{aligned}
$$

where $d_{t} \doteq \partial_{t}+\mathbf{v} \cdot \nabla$ is the convective derivative associated with velocity field $\mathbf{v} \doteq(\boldsymbol{\nabla} \theta-q \mathbf{A}) / m$, and

$$
\begin{gathered}
U_{\mathrm{SG}}=-\frac{q}{2 m} \eta^{\dagger}(\boldsymbol{\sigma} \cdot \mathbf{B}) \eta, \\
W_{\mathrm{P}}=\frac{(\boldsymbol{\nabla} a)^{2}}{2 m}+\frac{\mathcal{I}}{2 m}\left(\boldsymbol{\nabla} \eta^{\dagger}\right) \cdot(\boldsymbol{\nabla} \eta),
\end{gathered}
$$

where we made use of the fact that $\eta^{\dagger} \eta=1$. The term $U_{\mathrm{SG}}$ is the Stern-Gerlach energy. It can be expressed conveniently as $U_{\mathrm{SG}}=-(q / m) \mathbf{S} \cdot \mathbf{B}$, where

$$
\mathbf{S} \doteq \frac{1}{2} \eta^{\dagger} \boldsymbol{\sigma} \eta=\frac{1}{2}\left(\begin{array}{c}
\sin \zeta \cos \vartheta \\
\sin \zeta \sin \vartheta \\
\cos \zeta
\end{array}\right)
$$

is the spin vector, $S=1 / 2$. Expressing also the other terms through the four independent variables, $(\theta, \mathcal{I}, \vartheta, \zeta)$, one gets

$$
\begin{aligned}
\mathfrak{L}=-\mathcal{I}\left[\partial_{t} \theta\right. & +\frac{1}{2 m}(\nabla \theta-q \mathbf{A})^{2}+q \varphi \\
& \left.-\frac{1}{2}\left(d_{t} \vartheta\right) \cos \zeta-\frac{q}{m} \mathbf{S}(\vartheta, \zeta) \cdot \mathbf{B}\right]-W_{\mathrm{P}}
\end{aligned}
$$

$$
W_{\mathrm{P}}=\frac{(\boldsymbol{\nabla} \mathcal{I})^{2}}{8 m \mathcal{I}}+\frac{\mathcal{I}}{8 m}\left[(\nabla \zeta)^{2}+(\boldsymbol{\nabla} \vartheta)^{2}\right] .
$$

Hence, the following four ELEs are yielded. The first one is the ACT,

$$
\delta \theta: \quad \partial_{t} \mathcal{I}+\nabla \cdot(\mathcal{I} \mathbf{V})=0,
$$

where the flow velocity is given by $\mathbf{V}=\mathbf{v}+\mathbf{u}$, and

$$
\mathbf{u} \doteq-\frac{i}{2 m}\left[\eta^{\dagger}(\nabla \eta)-\left(\boldsymbol{\nabla} \eta^{\dagger}\right) \eta\right]=-\frac{1}{2 m}(\nabla \vartheta) \cos \zeta .
$$

The second ELE is a Hamilton-Jacobi equation,

$$
\begin{aligned}
\delta \mathcal{I}: \quad \partial_{t} \theta & +\frac{1}{2 m}(\boldsymbol{\nabla} \theta-q \mathbf{A})^{2}+q \varphi+Q-\frac{q}{m} \mathbf{S} \cdot \mathbf{B} \\
= & \frac{1}{2}\left(d_{t} \vartheta\right) \cos \zeta-\frac{1}{8 m}\left[(\boldsymbol{\nabla} \zeta)^{2}+(\boldsymbol{\nabla} \vartheta)^{2}\right] .
\end{aligned}
$$

Finally, the remaining two ELEs are

$$
\begin{array}{r}
\delta \vartheta: \quad \partial_{t}(\mathcal{I} \cos \zeta)+\nabla \cdot(\mathcal{I} \mathbf{v} \cos \zeta) \\
=\frac{1}{2 m} \nabla \cdot(\mathcal{I} \nabla \vartheta)+\frac{2 q \mathcal{I}}{m}\left(\partial_{\vartheta} \mathbf{S}\right) \cdot \mathbf{B}
\end{array}
$$

and

$$
\delta \zeta: \quad \mathcal{I}\left(d_{t} \vartheta\right) \sin \zeta=\frac{1}{2 m} \nabla \cdot(\mathcal{I} \nabla \zeta)+\frac{2 q \mathcal{I}}{m}\left(\partial_{\zeta} \mathbf{S}\right) \cdot \mathbf{B} .
$$

As shown in appendix, Eqs. (43)-(45) represent an alternative form of Takabayasi equations [21],

$$
\begin{aligned}
m\left(\partial_{t}+\right. & \mathbf{V} \cdot \boldsymbol{\nabla}) \mathbf{V}=q(\mathbf{E}+\mathbf{V} \times \mathbf{B})-\nabla Q \\
& +\frac{q}{m} S^{i}\left(\boldsymbol{\nabla} B_{i}\right)-\frac{1}{m \mathcal{I}} \partial_{j}\left[\mathcal{I}\left(\nabla S_{i}\right)\left(\partial^{j} S^{i}\right)\right]
\end{aligned}
$$




$$
\partial_{t} \mathbf{S}+(\mathbf{V} \cdot \nabla) \mathbf{S}=\frac{q}{m} \mathbf{S} \times \mathbf{B}+\frac{1}{m \mathcal{I}} \mathbf{S} \times \partial_{i}\left(\mathcal{I} \partial^{i} \mathbf{S}\right)
$$

(which were also studied recently in Refs. [30-35]). However, our equations have a number of advantages: (i) they have a manifestly Lagrangian form; (ii) the spin dynamics is described by two rather than three equations; (iii) $S=1 / 2$ is ensured irrespective of initial conditions.

The Lagrangian density for cold fluid is obtained by replacing $\mathcal{I}$ with $n$, as in Sec. III C, and by assuming that the real angles, which describe the particle spin, are about the same; i.e., $\vartheta_{q} \approx \vartheta$ and $\zeta_{q} \approx \zeta$, where $\vartheta$ and $\zeta$ are the corresponding average angles. Hence,

$$
\begin{aligned}
\mathfrak{L}=-n\left[\partial_{t} \theta\right. & +\frac{1}{2 m}(\boldsymbol{\nabla} \theta-q \mathbf{A})^{2}+q \varphi \\
& \left.-\frac{1}{2}\left(d_{t} \vartheta\right) \cos \zeta-\frac{q}{m} \mathbf{S}(\vartheta, \zeta) \cdot \mathbf{B}\right]-W_{\mathrm{P}}
\end{aligned}
$$

can be identified as a Lagrangian density of a cold fluid comprised of Pauli particles. The point-particle Lagrangian is derived much like in Sec. III B. We neglect the Bohm quantum potential and also assume that $\vartheta$ and $\zeta$ are approximately homogeneous in the regions in which $\mathcal{I}(t, \mathbf{x})$ is nonzero. Specifically, one obtains

$$
L=\mathbf{P} \cdot \dot{\mathbf{X}}+\frac{1}{2} \dot{\vartheta} \cos \zeta-H(t, \mathbf{X}, \mathbf{P}, \vartheta, \zeta),
$$

where the Hamiltonian $H$ is given by

$$
\begin{aligned}
H(t, \mathbf{X}, \mathbf{P}, \vartheta, \zeta) & =\frac{1}{2 m}(\mathbf{P}-q \mathbf{A}(t, \mathbf{X}))^{2} \\
& +q \varphi(t, \mathbf{X})-\frac{q}{m} \mathbf{S}(\vartheta, \zeta) \cdot \mathbf{B}(t, \mathbf{X})
\end{aligned}
$$

The corresponding ELEs are

$$
\begin{aligned}
\delta \mathbf{P}: & \dot{\mathbf{X}}=\partial_{\mathbf{P}} H(t, \mathbf{X}, \mathbf{P}, \vartheta, \zeta), \\
\delta \mathbf{X}: & \dot{\mathbf{P}}=-\partial_{\mathbf{X}} H(t, \mathbf{X}, \mathbf{P}, \vartheta, \zeta), \\
\delta \vartheta: & \dot{\zeta} \sin \zeta=-(2 q / m)\left(\partial_{\vartheta} \mathbf{S}\right) \cdot \mathbf{B}, \\
\delta \zeta: & \dot{\vartheta} \sin \zeta=+(2 q / m)\left(\partial_{\zeta} \mathbf{S}\right) \cdot \mathbf{B} .
\end{aligned}
$$

These equations also yield the following equation for $\mathbf{S}$,

$$
\dot{\mathbf{S}}=\frac{q}{m} \mathbf{S} \times \mathbf{B}(t, \mathbf{X}),
$$

as can be checked by direct substitution. Also, even without such calculation, the fact that $\dot{\mathbf{S}}$ is perpendicular to $\mathbf{S}$ is anticipated from the definition of the spin vector, Eq. (40), according to which $S$ must remain constant.

Notice that, within our approach, the spin precession equation flows directly from the VP. This distinguishes our approach from other calculations (e.g., in Refs. [36, 37]), where the equation for $\mathbf{S}$ is derived from a postulated Poisson bracket for the classical spin variables. We also notice that our Lagrangian can be represented in terms of complex variables, in a form akin to but different from those in Refs. [38-41]. This complex representation and also a detailed comparison with related point-particle models of spin electrons is discussed in a companion paper [15], where our results are extended to relativistic particles.

\section{KLEIN-GORDON PARTICLE}

It is instructive to discuss also Lagrangian densities of other types. For example, let us consider [42]

$$
\mathfrak{L}=-\frac{1}{2 m}\left[\left(D^{\mu} \psi\right)^{*}\left(D_{\mu} \psi\right)+m^{2} \psi^{*} \psi\right],
$$

which corresponds to a Klein-Gordon particle [43, 44], i.e., a relativistic spinless particle governed by

$$
\left(D_{\mu} D^{\mu}+m^{2}\right) \psi=0
$$

Here $D_{\mu} \doteq-i \partial_{\mu}-q A_{\mu}, A_{\mu}=A_{\mu}\left(x^{\nu}\right)$ is an electromagnetic four-vector potential ( $\operatorname{so} \varphi=-A_{0}$ ), and $\psi$ is complex scalar function. [The factor $(2 m)^{-1}$ in Eq. (56) is added to ensure that $\psi$ is normalized such that $\psi^{*} \psi$ relates to the action density most transparently; see below.] Let us represent the state function as

$$
\psi=e^{i \theta} \sqrt{\mathcal{I}_{0}}
$$

and adopt the semiclassical limit, like in Sec. III. Then,

$$
\mathfrak{L} \approx \frac{\mathcal{I}_{0}}{2 m}\left[\left(\partial_{t} \theta+q \varphi\right)^{2}-(\boldsymbol{\nabla} \theta-q \mathbf{A})^{2}-m^{2}\right] .
$$

This has the form of Whitham's Lagrangian density [10],

$$
\mathfrak{L}=\mathfrak{D}(\underbrace{-\partial_{t} \theta}_{\mathcal{E}}, \underbrace{\nabla \theta}_{\mathbf{p}} ; t, \mathbf{x}) \mathcal{I}_{0}
$$

in which

$$
\mathcal{I} \doteq \mathcal{I}_{0} \partial_{\mathcal{E}} \mathfrak{D}(\mathcal{E}, \mathbf{p} ; t, \mathbf{x})=\gamma \mathcal{I}_{0}
$$

serves as the action density, and $\gamma \doteq(\mathcal{E}-q \varphi) / m$. This yields the ACT and a Hamilton-Jacobi equation,

$$
\begin{array}{ll}
\delta \theta: & \partial_{t} \mathcal{I}+\nabla \cdot(\mathcal{I} \mathbf{v})=0 \\
\delta \mathcal{I}: & \left(\partial_{t} \theta+q \varphi\right)^{2}=(\boldsymbol{\nabla} \theta-q \mathbf{A})^{2}+m^{2},
\end{array}
$$

where the flow velocity $\mathbf{v}$ is given by

$$
\mathbf{v} \doteq \frac{\boldsymbol{\nabla} \theta-q \mathbf{A}}{m \gamma}
$$

Hence one may recognize $\gamma$ as the Lorentz factor [because $\mathcal{E}$ serves as the canonical energy (Sec. III) and, from Eq. (63), it is seen that $\mathcal{E}-q \varphi$ is the kinetic energy], so $\mathcal{I}_{0}$ is recognized as the proper action density.

From Eq. (63), one gets

$$
-\partial_{t} \theta= \pm \sqrt{(\boldsymbol{\nabla} \theta-q \mathbf{A})^{2}+m^{2}}+q \varphi .
$$


To ensure that the previously adopted semiclassical approximation is satisfied, one of the two solutions must be excluded. We will consider positive-energy particles, i.e., assume the plus sign. Then the wave is of a scalar type, and, as it is well known [25], Whitham's Lagrangian density can be rewritten in the Hayes's form (8); namely,

$$
\mathfrak{L} \approx-\mathcal{I}\left\{\partial_{t} \theta+\sqrt{[\mathbf{p}-q \mathbf{A}(t, \mathbf{x})]^{2}+m^{2}}+q \varphi(t, \mathbf{x})\right\} .
$$

As in the previous sections, the Lagrangian density of a cold (yet now relativistic) classical fluid is obtained by replacing $\mathcal{I}$ with $n$. The corresponding ELEs coincide with Eqs. (22) and (23), and taking the gradient of the latter leads to the well known relativistic momentum equation,

$$
\left[\partial_{t}+(\mathbf{v} \cdot \boldsymbol{\nabla})\right](m \gamma \mathbf{v})=q(\mathbf{E}+\mathbf{v} \times \mathbf{B}) .
$$

Also, the classical point-particle motion is automatically seen to be governed by Eqs. (17) and (18) with

$$
H(t, \mathbf{X}, \mathbf{P})=\sqrt{[\mathbf{P}-q \mathbf{A}(t, \mathbf{X})]^{2}+m^{2}}+q \varphi(t, \mathbf{X}) .
$$

Finally, let us discuss the interactions of particles with self-consistent fields. The Lagrangian density $\mathfrak{L}_{\Sigma}$ of such interactions is obtained, as usual, by adding to $\mathfrak{L}$ the Lagrangian density of the vacuum field [45, Sec. 11.5],

$$
\mathfrak{L}_{\mathrm{EM}}=\frac{1}{8 \pi}\left(-\boldsymbol{\nabla} \varphi-\partial_{t} \mathbf{A}\right)^{2}-\frac{|\boldsymbol{\nabla} \times \mathbf{A}|^{2}}{8 \pi} \equiv \frac{E^{2}-B^{2}}{8 \pi},
$$

where $\mathbf{E}$ and $\mathbf{B}$ are electric and magnetic fields. This gives

$$
\mathfrak{L}_{\Sigma}=\mathfrak{L}_{\mathrm{EM}}-\sum_{s} n_{s}\left[\partial_{t} \theta_{s}+H_{s}\left(t, \mathbf{x}, \nabla \theta_{s}\right)\right],
$$

where the sum is taken over all species. In particular, substituting here the Hamiltonian (68) automatically yields the well-known Chen-Sudan Lagrangian density of cold relativistic plasma [20]. It is to be noted that, in the form (69), the obtained $\mathfrak{L}_{\Sigma}$ can be treated as the Lagrangian density of warm plasma too, if particles with different energies are treated as different species; for example, see Ref. [11].

\section{DISCUSSION}

We showed that classical VPs can be deduced from quantum VPs, which are well known, via formal reparameterization of the latter. Such reparameterization is possible without appealing to dynamical equations and without invoking any assumptions other than classicality. Classical-fluid Lagrangians flow as the semiclassical limit of the FQL, and the point-particle Lagrangians are then yielded as corollaries for narrow but otherwise arbitrary wave packets. We explicitly performed these calculations for several commonly known systems, namely, Schrödinger, Pauli, and Klein-Gordon particles.
We showed that, for instance, the expression for the Bohm quantum potential, the so-called Weizsäcker correction, the Madelung equations, and the Chen-Sudan Lagrangian density all emerge naturally within our unifying theory as special cases. We also obtained an alternative, manifestly Lagrangian representation of Takabayasi equations for a Pauli particle. Our model yields the dynamics of the spin vector $\mathbf{S}$ directly from the VP and employs two, rather than three, equations; hence, $S=1 / 2$ is ensured irrespective of initial conditions.

Our results can be viewed as a generalization of and a complement to reparameterizations of the FQL that were reported in literature earlier [14]. In addition to this, the new approach has several important applications. First of all, it enables a first principle classical Lagrangian description of most general, relativistic vector particles such as a Dirac electron, which is a longstanding problem (see, e.g., Ref. [46]). Second, the same theory is applicable as is to a GO description of classical vector waves, since the fundamental Lagrangian of classical waves is identical to the FQL [22]. This allows accounting for polarization effects and mode conversion directly in ray equations. This also allows to extend the recent studies of ponderomotive forces on scalar waves and particles [12] to more general, vector waves and particles. Third, calculations of even purely classical dynamics can be simplified through the application of quantum Lagrangians. This is because, in contrast to classical equations for point particles, semiclassical equations are linear and thus sometimes are easier to work with. These and other applications of the theory presented here will be discussed in follow-up papers, including Ref. [15].

The authors thank J. W. Burby and N. J. Fisch for valuable discussions. The work was supported by the NNSA SSAA Program through DOE Research Grant No. DE274-FG52-08NA28553, by the U.S. DOE through Contract No. DE-AC02-09CH11466, and by the U.S. DOD NDSEG Fellowship through Contract No. FA955011-C-0028.

\section{Appendix A: Takabayasi equations}

In this appendix, we show that the ELEs derived in Sec. V for a Pauli particle agree with Takabayasi equations (46) and (47).

\section{Momentum equation}

To derive the momentum equation, we begin by rewriting the "spin stress" introduced by Takabayasi [21],

$$
\Pi \doteq-\frac{1}{m \mathcal{I}} \partial_{j}\left[\mathcal{I}\left(\nabla S_{i}\right)\left(\partial^{j} S^{i}\right)\right]
$$


in Eq. (46). Specifically, by substituting Eq. (40) for $\mathbf{S}$ in Eq. (A1), one can write

$$
\Pi=-\frac{1}{4 m \mathcal{I}} \partial_{j}\left[\mathcal{I}(\nabla \zeta)\left(\partial^{j} \zeta\right)+\mathcal{I} \sin ^{2} \zeta(\nabla \vartheta)\left(\partial^{j} \vartheta\right)\right]
$$

Also, let us use Eq. (45) to express $d_{t} \vartheta$ and substitute the result in the right hand side of Eq. (43). Taking the gradient of the resulting equation yields

$$
\begin{aligned}
m d_{t} \mathbf{v}=q(\mathbf{E}+\mathbf{v} \times \mathbf{B})-\boldsymbol{\nabla} Q+\frac{q}{m} \boldsymbol{\nabla}\left[\mathbf{S} \cdot \mathbf{B}+\cot \zeta\left(\partial_{\zeta} \mathbf{S}\right) \cdot \mathbf{B}\right] & \\
& +\boldsymbol{\nabla}\left[\frac{\cot \zeta}{4 m \mathcal{I}} \boldsymbol{\nabla} \cdot(\mathcal{I} \nabla \zeta)\right]-\frac{1}{8 m} \boldsymbol{\nabla}\left[(\boldsymbol{\nabla} \zeta)^{2}+(\boldsymbol{\nabla} \vartheta)^{2}\right] .
\end{aligned}
$$

Proceeding with the calculation of the conservation equation for $m \mathbf{u}$, one obtains

$$
\begin{aligned}
m \partial_{t} \mathbf{u}= & -\frac{1}{2} \partial_{t}[(\boldsymbol{\nabla} \vartheta) \cos \zeta] \\
= & -\frac{1}{2}(\boldsymbol{\nabla} \vartheta) \partial_{t}(\cos \zeta)-\frac{1}{2} \cos \zeta \boldsymbol{\nabla}\left(\partial_{t} \vartheta\right) \\
= & -(\boldsymbol{\nabla} \vartheta)\left[-\frac{1}{2}(\mathbf{v} \cdot \boldsymbol{\nabla}) \cos \zeta+\frac{\cos \zeta}{2 \mathcal{I}} \boldsymbol{\nabla} \cdot(\mathcal{I} \mathbf{u})+\frac{1}{4 m \mathcal{I}} \boldsymbol{\nabla} \cdot(\mathcal{I} \nabla \vartheta)+\frac{q}{m}\left(\partial_{\vartheta} \mathbf{S}\right) \cdot \mathbf{B}\right] \\
& -\cos \zeta \boldsymbol{\nabla}\left[-\frac{1}{2}(\mathbf{v} \cdot \boldsymbol{\nabla}) \vartheta+\frac{1}{4 m \mathcal{I} \sin \zeta} \boldsymbol{\nabla} \cdot(\mathcal{I} \boldsymbol{\nabla} \zeta)+\frac{q}{m \sin \zeta}\left(\partial_{\zeta} \mathbf{S}\right) \cdot \mathbf{B}\right] \\
= & -m(\mathbf{v} \cdot \boldsymbol{\nabla}) \mathbf{u}-m\left(\boldsymbol{\nabla} v_{i}\right) u^{i}+\frac{m \mathbf{u}}{\mathcal{I}} \boldsymbol{\nabla} \cdot(\mathcal{I} \mathbf{u})-\frac{(\boldsymbol{\nabla} \vartheta)}{4 m \mathcal{I}} \boldsymbol{\nabla} \cdot(\mathcal{I} \boldsymbol{\nabla} \vartheta) \\
& -\frac{q}{m}\left[\left(\partial_{\vartheta} \mathbf{S}\right) \cdot \mathbf{B}\right] \boldsymbol{\nabla} \vartheta-\cos \zeta \boldsymbol{\nabla}\left[\frac{q}{m \sin \zeta}\left(\partial_{\zeta} \mathbf{S}\right) \cdot \mathbf{B}\right]-\cos \zeta \boldsymbol{\nabla}\left[\frac{1}{4 m \mathcal{I} \sin \zeta} \boldsymbol{\nabla} \cdot(\mathcal{I} \nabla \zeta)\right] \\
= & -m(\mathbf{v} \cdot \boldsymbol{\nabla}) \mathbf{u}-m(\mathbf{u} \cdot \boldsymbol{\nabla}) \mathbf{v}+q \mathbf{u} \times \mathbf{B}+\frac{\cos \zeta}{4 m \mathcal{I}}(\boldsymbol{\nabla} \vartheta) \boldsymbol{\nabla} \cdot[(\boldsymbol{\nabla} \vartheta) \mathcal{I} \cos \zeta]-\frac{(\boldsymbol{\nabla} \vartheta)}{4 m \mathcal{I}} \boldsymbol{\nabla} \cdot(\mathcal{I} \nabla \vartheta) \\
& -\frac{q}{m}\left[\left(\partial_{\vartheta} \mathbf{S}\right) \cdot \mathbf{B}\right](\boldsymbol{\nabla} \vartheta)-\cos \zeta \boldsymbol{\nabla}\left[\frac{q}{m \sin \zeta}\left(\partial_{\zeta} \mathbf{S}\right) \cdot \mathbf{B}\right]-\cos \zeta \boldsymbol{\nabla}\left[\frac{1}{4 m \mathcal{I} \sin \zeta} \boldsymbol{\nabla} \cdot(\mathcal{I} \nabla \zeta)\right]
\end{aligned}
$$

where we used Eqs. (44) and (45). Let us add Eqs. (A3) and (A4) and also add $(\mathbf{u} \cdot \boldsymbol{\nabla}) \mathbf{u}$ to both sides in order to complete the convective derivative $\hat{\mathcal{D}} \doteq \partial_{t}+\mathbf{V} \cdot \boldsymbol{\nabla}$. One then gets

$$
\begin{aligned}
m \hat{\mathcal{D}} \mathbf{V}= & q(\mathbf{E}+\mathbf{V} \times \mathbf{B})-\boldsymbol{\nabla} Q+\frac{q}{m} S^{i}\left(\boldsymbol{\nabla} B_{i}\right)+m(\mathbf{u} \cdot \boldsymbol{\nabla}) \mathbf{u}-\frac{(\boldsymbol{\nabla} \zeta)}{4 m \mathcal{I}} \boldsymbol{\nabla} \cdot(\mathcal{I} \boldsymbol{\nabla} \zeta)-\frac{1}{8 m} \boldsymbol{\nabla}\left[(\boldsymbol{\nabla} \zeta)^{2}\right] \\
& -\frac{\sin ^{2} \zeta}{4 m \mathcal{I}}(\boldsymbol{\nabla} \vartheta) \boldsymbol{\nabla} \cdot(\mathcal{I} \boldsymbol{\nabla} \vartheta)-\frac{\sin ^{2} \zeta}{4 m}(\boldsymbol{\nabla} \vartheta \cdot \boldsymbol{\nabla})(\boldsymbol{\nabla} \vartheta)-\frac{\sin \zeta \cos \zeta}{2 m}(\boldsymbol{\nabla} \vartheta)[(\boldsymbol{\nabla} \vartheta) \cdot(\boldsymbol{\nabla} \zeta)] \\
= & q(\mathbf{E}+\mathbf{V} \times \mathbf{B})-\boldsymbol{\nabla} Q+\frac{q}{m} S^{i}\left(\boldsymbol{\nabla} B_{i}\right)-\frac{1}{4 m \mathcal{I}} \partial_{j}\left[\mathcal{I}(\boldsymbol{\nabla} \zeta)\left(\partial^{j} \zeta\right)+\mathcal{I} \sin ^{2} \zeta(\boldsymbol{\nabla} \vartheta)\left(\partial^{j} \vartheta\right)\right] \\
= & q(\mathbf{E}+\mathbf{V} \times \mathbf{B})-\boldsymbol{\nabla} Q+\frac{q}{m} S^{i}\left(\boldsymbol{\nabla} B_{i}\right)-\frac{1}{m \mathcal{I}} \partial_{j}\left[\mathcal{I}\left(\nabla S_{i}\right)\left(\partial^{j} S^{i}\right)\right],
\end{aligned}
$$

where we substituted Eq. (A2) at the end. Hence, we see that the ELEs derived in Sec. V lead to Eq. (46).

\section{Spin equation}

To derive the spin equation, let us start by re-expressing the "spin torque" introduced by Takabayasi [21],

$$
\mathbf{M} \doteq \frac{1}{m \mathcal{I}}\left[\mathbf{S} \times \partial_{j}\left(\mathcal{I} \partial^{j} \mathbf{S}\right)\right]
$$


in terms of $\vartheta$ and $\zeta$. Specifically, we get

$$
\begin{aligned}
& M_{x}=\frac{1}{m \mathcal{I}} S_{y} \nabla \cdot\left(\mathcal{I} \nabla S_{z}\right)-\frac{1}{m \mathcal{I}} S_{z} \nabla \cdot\left(\mathcal{I} \nabla S_{y}\right) \\
& =\frac{1}{4 m \mathcal{I}} \sin \zeta \sin \vartheta \nabla \cdot[\mathcal{I} \nabla(\cos \zeta)]-\frac{1}{4 m \mathcal{I}} \cos \zeta \nabla \cdot[\mathcal{I} \nabla(\sin \zeta \sin \vartheta)] \\
& =-\frac{1}{4 m \mathcal{I}} \sin \zeta \sin \vartheta \boldsymbol{\nabla} \cdot[\mathcal{I}(\boldsymbol{\nabla} \zeta) \sin \zeta]-\frac{1}{4 m \mathcal{I}} \cos \zeta \boldsymbol{\nabla} \cdot[\mathcal{I}(\boldsymbol{\nabla} \zeta) \cos \zeta \sin \vartheta]-\frac{1}{4 m \mathcal{I}} \cos \zeta \boldsymbol{\nabla} \cdot[\mathcal{I}(\boldsymbol{\nabla} \vartheta) \sin \zeta \cos \vartheta] \\
& =\frac{\sin \vartheta}{4 m}\left[(\nabla \vartheta)^{2} \sin \zeta \cos \zeta-\frac{1}{\mathcal{I}} \nabla \cdot(\mathcal{I} \nabla \zeta)\right] \\
& -\frac{1}{4 m}(\boldsymbol{\nabla} \zeta)^{2} \sin \zeta \cos \zeta \sin \vartheta-\frac{1}{4 m} \cos \zeta(\boldsymbol{\nabla} \zeta) \cdot \boldsymbol{\nabla}(\cos \zeta \sin \vartheta)-\frac{1}{4 m \mathcal{I}} \cos \zeta \cos \vartheta \boldsymbol{\nabla} \cdot[\mathcal{I}(\boldsymbol{\nabla} \vartheta) \sin \zeta] \\
& =\frac{\sin \vartheta}{4 m}\left[(\boldsymbol{\nabla} \vartheta)^{2} \sin \zeta \cos \zeta-\frac{1}{\mathcal{I}} \boldsymbol{\nabla} \cdot(\mathcal{I} \nabla \zeta)\right]-\frac{1}{4 m \mathcal{I}} \cot \zeta \cos \vartheta \nabla \cdot\left[\mathcal{I}(\boldsymbol{\nabla} \vartheta) \sin ^{2} \zeta\right] \\
& M_{y}=\frac{1}{m \mathcal{I}} S_{z} \nabla \cdot\left(\mathcal{I} \nabla S_{x}\right)-\frac{1}{m \mathcal{I}} S_{x} \nabla \cdot\left(\mathcal{I} \nabla S_{z}\right) \\
& =\frac{1}{4 m \mathcal{I}} \cos \zeta \nabla \cdot[\mathcal{I} \nabla(\sin \zeta \cos \vartheta)]-\frac{1}{4 m \mathcal{I}} \sin \zeta \cos \vartheta \nabla \cdot[\mathcal{I} \nabla(\cos \zeta)] \\
& =\frac{1}{4 m \mathcal{I}} \cos \zeta \boldsymbol{\nabla} \cdot[\mathcal{I}(\boldsymbol{\nabla} \zeta) \cos \zeta \cos \vartheta]-\frac{1}{4 m \mathcal{I}} \cos \zeta \boldsymbol{\nabla} \cdot[\mathcal{I}(\boldsymbol{\nabla} \vartheta) \sin \zeta \sin \vartheta]+\frac{1}{4 m \mathcal{I}} \sin \zeta \cos \vartheta \boldsymbol{\nabla} \cdot[\mathcal{I}(\boldsymbol{\nabla} \zeta) \cos \zeta] \\
& =-\frac{\cos \vartheta}{4 m}\left[(\boldsymbol{\nabla} \vartheta)^{2} \sin \zeta \cos \zeta-\frac{1}{\mathcal{I}} \boldsymbol{\nabla} \cdot(\mathcal{I} \boldsymbol{\nabla} \zeta)\right]-\frac{1}{4 m \mathcal{I}} \cot \zeta \sin \vartheta \nabla \cdot\left[\mathcal{I}(\boldsymbol{\nabla} \vartheta) \sin ^{2} \zeta\right], \\
& M_{z}=\frac{1}{m \mathcal{I}} S_{x} \boldsymbol{\nabla} \cdot\left(\mathcal{I} \nabla S_{y}\right)-\frac{1}{m \mathcal{I}} S_{y} \nabla \cdot\left(\mathcal{I} \nabla S_{x}\right) \\
& =\frac{1}{4 m \mathcal{I}} \sin \zeta \cos \vartheta \nabla \cdot[\mathcal{I} \nabla(\sin \zeta \sin \vartheta)]-\frac{1}{4 m \mathcal{I}} \sin \zeta \sin \vartheta \nabla \cdot[\mathcal{I} \nabla(\cos \zeta \cos \vartheta)] \\
& =\frac{\sin \zeta \cos \zeta}{2 m}(\boldsymbol{\nabla} \zeta) \cdot(\boldsymbol{\nabla} \vartheta)+\frac{\sin ^{2} \zeta}{4 m \mathcal{I}}(\boldsymbol{\nabla} \mathcal{I}) \cdot(\boldsymbol{\nabla} \vartheta)+\frac{\sin ^{2} \zeta}{4 m}\left(\boldsymbol{\nabla}^{2} \vartheta\right) \\
& =\frac{1}{4 m \mathcal{I}} \boldsymbol{\nabla} \cdot\left[\mathcal{I}(\boldsymbol{\nabla} \vartheta) \sin ^{2} \zeta\right] \text {. }
\end{aligned}
$$

Hence, the conservation equations for the spin vector $\mathbf{S}$ can be expressed as follows:

$$
\begin{aligned}
\hat{\mathcal{D}} S_{x}= & \frac{1}{2} \partial_{t}(\sin \zeta \cos \vartheta)+\frac{1}{2}(\mathbf{V} \cdot \boldsymbol{\nabla})(\sin \zeta \cos \vartheta) \\
= & \frac{1}{2} \cos \zeta \cos \vartheta \hat{\mathcal{D}} \zeta-\frac{1}{2} \sin \zeta \sin \vartheta \hat{\mathcal{D}} \vartheta \\
= & -\cot \zeta \cos \vartheta \hat{\mathcal{D}} S_{z}-\frac{1}{2} \sin \vartheta \sin \zeta \hat{\mathcal{D}} \vartheta \\
= & -\cot \zeta \cos \vartheta\left\{\frac{1}{4 m \mathcal{I}} \boldsymbol{\nabla} \cdot\left[\mathcal{I}(\boldsymbol{\nabla} \vartheta) \sin ^{2} \zeta\right]+\frac{q}{m}\left(\partial_{\vartheta} \mathbf{S}\right) \cdot \mathbf{B}\right\} \\
& -\sin \vartheta\left[\frac{1}{2} \sin \zeta(\mathbf{u} \cdot \boldsymbol{\nabla}) \vartheta+\frac{1}{4 m \mathcal{I}} \boldsymbol{\nabla} \cdot(\mathcal{I} \boldsymbol{\nabla} \zeta)+\frac{q}{m}\left(\partial_{\zeta} \mathbf{S}\right) \cdot \mathbf{B}\right] \\
= & -\cot \zeta \cos \vartheta\left[\frac{1}{4 m \mathcal{I}} \boldsymbol{\nabla} \cdot\left[\mathcal{I}(\boldsymbol{\nabla} \vartheta) \sin ^{2} \zeta\right]+\frac{q}{m}\left(\partial_{\vartheta} \mathbf{S}\right) \cdot \mathbf{B}\right] \\
& -\sin \vartheta\left[-\frac{1}{4 m}(\boldsymbol{\nabla} \vartheta)^{2} \sin \zeta \cos \zeta+\frac{1}{4 m \mathcal{I}} \boldsymbol{\nabla} \cdot(\mathcal{I} \boldsymbol{\nabla} \zeta)+\frac{q}{m}\left(\partial_{\zeta} \mathbf{S}\right) \cdot \mathbf{B}\right] \\
= & -\frac{1}{4 m \mathcal{I}} \cot \zeta \cos \vartheta \nabla \cdot\left[\mathcal{I}(\nabla \vartheta) \sin ^{2} \zeta\right]+\sin \vartheta\left[\frac{1}{4 m}(\boldsymbol{\nabla} \vartheta)^{2} \sin \zeta \cos \zeta-\frac{1}{4 m \mathcal{I}} \boldsymbol{\nabla} \cdot(\mathcal{I} \boldsymbol{\nabla} \zeta)\right]+\frac{q}{m}(\mathbf{S} \times \mathbf{B})_{x} \\
= & \frac{1}{m \mathcal{I}}\left[\mathbf{S} \times \partial_{j}\left(\mathcal{I} \partial^{j} \mathbf{S}\right)\right]_{x}+\frac{q}{m}(\mathbf{S} \times \mathbf{B})_{x},
\end{aligned}
$$


where we substituted Eq. (A7); also,

$$
\begin{aligned}
\hat{\mathcal{D}} S_{y}= & \frac{1}{2} \partial_{t}(\sin \zeta \sin \vartheta)+\frac{1}{2}(\mathbf{V} \cdot \boldsymbol{\nabla})(\sin \zeta \sin \vartheta) \\
= & \frac{1}{2} \cos \zeta \sin \vartheta \hat{\mathcal{D}} \zeta+\frac{1}{2} \sin \zeta \cos \vartheta \hat{\mathcal{D}} \vartheta \\
= & -\cot \zeta \sin \vartheta \hat{\mathcal{D}} S_{z}+\frac{1}{2} \cos \vartheta \sin \zeta \hat{\mathcal{D}} \vartheta \\
= & -\cot \zeta \sin \vartheta\left\{\frac{1}{4 m \mathcal{I}} \boldsymbol{\nabla} \cdot\left[\mathcal{I}(\boldsymbol{\nabla} \vartheta) \sin ^{2} \zeta\right]+\frac{q}{m}\left(\partial_{\vartheta} \mathbf{S}\right) \cdot \mathbf{B}\right\} \\
& +\cos \vartheta\left[\frac{1}{2} \sin \zeta(\mathbf{u} \cdot \boldsymbol{\nabla}) \vartheta+\frac{1}{4 m \mathcal{I}} \boldsymbol{\nabla} \cdot(\mathcal{I} \boldsymbol{\nabla} \zeta)+\frac{q}{m}\left(\partial_{\zeta} \mathbf{S}\right) \cdot \mathbf{B}\right] \\
= & -\frac{1}{4 m \mathcal{I}} \cot \zeta \sin \vartheta \boldsymbol{\nabla} \cdot\left[\mathcal{I}(\boldsymbol{\nabla} \vartheta) \sin ^{2} \zeta\right]-\cos \vartheta\left[\frac{1}{4 m}(\boldsymbol{\nabla} \vartheta)^{2} \sin \zeta \cos \zeta-\frac{1}{4 m \mathcal{I}} \boldsymbol{\nabla} \cdot(\mathcal{I} \boldsymbol{\nabla} \zeta)\right]+\frac{q}{m}(\mathbf{S} \times \mathbf{B})_{y} \\
= & \frac{1}{m \mathcal{I}}\left[\mathbf{S} \times \partial_{j}\left(\mathcal{I} \partial^{j} \mathbf{S}\right)\right]_{y}+\frac{q}{m}(\mathbf{S} \times \mathbf{B})_{y},
\end{aligned}
$$

where we substituted Eq. (A8). Finally, the equation for $S_{z}$ is obtained directly from Eq. (44),

$$
\mathcal{I}\left[\partial_{t} S_{z}+(\mathbf{v} \cdot \nabla) S_{z}\right]+S_{z}\left[\partial_{t} \mathcal{I}+\nabla \cdot(\mathcal{I} \mathbf{v})\right]=\frac{1}{4 m} \nabla \cdot(\mathcal{I} \nabla \vartheta)+\frac{q \mathcal{I}}{m}\left(\partial_{\vartheta} \mathbf{S}\right) \cdot \mathbf{B} .
$$

Using the ACT, one then gets

$$
\begin{aligned}
\hat{\mathcal{D}} S_{z} & =\frac{1}{\mathcal{I}}\left[\mathcal{I}(\mathbf{u} \cdot \boldsymbol{\nabla}) S_{z}+S_{z} \boldsymbol{\nabla} \cdot(\mathcal{I} \mathbf{u})\right]+\frac{1}{4 m \mathcal{I}} \boldsymbol{\nabla} \cdot(\mathcal{I} \nabla \vartheta)+\frac{q}{m}\left(\partial_{\vartheta} \mathbf{S}\right) \cdot \mathbf{B} \\
& =\frac{1}{\mathcal{I}} \boldsymbol{\nabla} \cdot\left(S_{z} \mathcal{I} \mathbf{u}\right)+\frac{1}{4 m \mathcal{I}} \boldsymbol{\nabla} \cdot(\mathcal{I} \nabla \vartheta)+\frac{q}{m}\left(\partial_{\vartheta} \mathbf{S}\right) \cdot \mathbf{B} \\
& =\frac{1}{4 m \mathcal{I}} \boldsymbol{\nabla} \cdot\left[\mathcal{I}(\boldsymbol{\nabla} \vartheta) \sin ^{2} \zeta\right]+\frac{q}{m}(\mathbf{S} \times \mathbf{B})_{z} \\
& =\frac{1}{m \mathcal{I}}\left[\mathbf{S} \times \partial_{j}\left(\mathcal{I} \partial^{j} \mathbf{S}\right)\right]_{z}+\frac{q}{m}(\mathbf{S} \times \mathbf{B})_{z},
\end{aligned}
$$

where we substituted Eq. (A9). Considered together as a vector equation, Eqs. (A10)-(A13) coincide with Eq. (47).

[1] H. Feldmeier and J. Schnack, Molecular dynamics for fermions, Rev. Mod. Phys. 72, 655 (2000).

[2] P. E. Grabowski, A review of wave packet molecular $d y$ namics, in Frontiers and Challenges in Warm Dense Matter, edited by F. Graziani, M. P. Desjarlais, R. Redmer, and S. B. Trickey (Springer, New York, 2014).

[3] R. L. Seliger and G. B. Whitham, Variational principles in continuum mechanics, Proc. R. Soc. A 305, 1 (1968).

[4] P. J. Morrison, Hamiltonian description of the ideal fluid, Rev. Mod. Phys. 70, 467 (1998).

[5] A. J. Brizard, Variational principles for reduced plasma physics, J. Phys. Conf. Ser. 169, 012003 (2009).

[6] J. P. Friedberg, Ideal Magnetohydrodynamics (Plenum Press, New York, 1987).

[7] G. B. Whitham, Linear and Nonlinear Waves (Wiley, New York, 1974).

[8] E. R. Tracy, A. J. Brizard, A. S. Richardson, and A. N. Kaufman, Ray Tracing and Beyond: Phase Space Methods in Plasma Wave Theory (Cambridge University
Press, New York, 2014).

[9] J. D. Joannopoulos, S. G. Johnson, J. N. Winn, and R. D. Meade, Photonic Crystals: Molding the Flow of Light (Princeton Univ. Press, Princeton, 2008), second edition.

[10] I. Y. Dodin and N. J. Fisch, Axiomatic geometrical optics, Abraham-Minkowski controversy, and photon properties derived classically, Phys. Rev. A 86, 053834 (2012).

[11] I. Y. Dodin, On variational methods in the physics of plasma waves, Fusion Sci. Tech. 65, 54 (2014).

[12] I. Y. Dodin and N. J. Fisch, Ponderomotive forces on waves in modulated media, Phys. Rev. Lett. 112, 205002 (2014).

[13] For given dynamic equations, a Lagrangian is usually guessed or constructed using Lagrange multipliers [3], neither of which methods is universal. The existing methods of deriving classical variational principles from quantum mechanics [14] are also limited, as we discuss below.

[14] For example, see Refs. [1, 2] and also G. Sundaram and 
Q. Niu, Phys. Rev. B 59, 14915 (1999).

[15] D. E. Ruiz and I. Y. Dodin, Lagrangian geometrical optics of nonadiabatic vector waves and spin particles, arXiv:1503.07829.

[16] D. Bohm, A suggested interpretation of the quantum theory in terms of "hidden variables". I, Phys. Rev. 85, 166 (1952).

[17] D. Bohm, A suggested interpretation of the quantum theory in terms of "hidden" variables. II, Phys. Rev. 85, 180 (1952).

[18] C. F. Von Weizsäcker, Zur Theorie der Kernmassen, Z. Phys. 96, 431 (1935).

[19] E. Madelung, Quantum theory in hydrodynamical form, Z. Phys. 40, 322 (1927).

[20] X. L. Chen and R. N. Sudan, Two-dimensional selffocusing of short intense laser pulse in underdense plasma, Phys. Fluids B 5, 1336 (1993).

[21] T. Takabayasi, The vector representation of spinning particle in the quantum theory, I, Prog. Theor. Phys. 14, 283 (1955).

[22] I. Y. Dodin, Geometric view on noneikonal waves, Phys. Lett. A 378, 1598 (2014).

[23] Another commonly used form of the FQL is $\mathfrak{L}=$ $\psi^{\dagger}\left(i \partial_{t} \psi-\hat{H} \psi\right)[1]$. This formula differs from Eq. (3) only by a perfect time derivative and thus is physically equivalent, i.e., leads to the same ELEs. Here we prefer Eq. (3), since the corresponding $\mathfrak{L}$ is real.

[24] Here we assumed that the particle has positive energy. Otherwise, signs will be different, because the scalar product in the $\psi$ space is not positively defined [22].

[25] W. D. Hayes, Group velocity and nonlinear dispersive wave propagation, Proc. R. Soc. Lond. A 332, 199 (1973).

[26] L. D. Landau and E. M. Lifshitz, Mechanics (Butterworth-Heinemann, Oxford, 1976).

[27] See also Refs. [3, 47] for comparison. Notably, the added term must be nonlinear in the density to introduce nontrivial thermal effects.

[28] D. Michta, F. Graziani, and M. Bonitz, Quantum hydrodynamics for plasmas - a Thomas-Fermi theory perspective, arXiv:1503.04037.

[29] W. Pauli, Zur Quantenmechanik des magnetischen Elektrons, Z. Phys. 43, 601 (1927).

[30] M. Stefan, J. Zamanian, G. Brodin, A. P. Misra, and M. Marklund, Ponderomotive force due to the intrinsic spin in extended fluid and kinetic models, Phys. Rev. E 83, 036410 (2011).

[31] G. Brodin, J. Lundin, J. Zamanian, and M Stefan, Nonlinear wave interaction and spin models in the magneto- hydrodynamic regime, New J. Phys. 13, 083017 (2011).

[32] J. Zamanian, M. Stefan, M. Marklund, and G. Brodin, From extended phase space dynamics to fluid theory, Phys. Plasmas 17, 102109 (2010).

[33] G. Brodin and M. Marklund, Spin magnetohydrodynamics, New J. Physics 9, 277 (2007).

[34] M. Marklund and G. Brodin, Dynamics of spin-1/2 quantum plasmas, Phys. Rev. Lett. 98, 025001 (2007).

[35] A related variational theory for spin-1/2 magnetohydrodynamics was also proposed recently in M. Lingam, Phys. Plasmas 22, 022124 (2015). However, in contrast with our formulation, which deduces the fluid Lagrangian from first principles, the mentioned theory is largely heuristic.

[36] Ya. S. Derbenev and A. M. Kondratenko, Polarization kinetics of particles in storage rings, Zh. Eksp. Tear. Fiz. 64, 1918 (1973) [Sov. Phys. JETP 37, 968 (1973)].

[37] K. Heinemann, On Stern-Gerlach forces allowed by special relativity and the special case of the classical spinning particle of Derbenev-Kondratenko, arXiv:physics/9611001v1.

[38] A. O. Barut and N. Zanghi, Classical model of the Dirac electron, Phys. Rev. Lett. 52, 2009 (1984).

[39] A. O. Barut and W. Thacker, Covariant generalization of the Zitterbewegung of the electron and its $S Q(4,2)$ and $S G(3,2)$ internal algebras, Phys. Rev. D 31, 1386 (1985).

[40] A. O. Barut, C. Önem, and N. Ünal, The classical relativistic two-body problem with spin and self-interactions, J. Phys. A: Math. Gen. 23, 1113 (1990).

[41] A. O. Barut and M. G. Cruz, Classical relativistic spinning particle with anomalous magnetic moment: the precession of spin, J. Phys. A: Math. Gen. 26, 6499 (1993).

[42] In fact, Eq. (57) is also derivable from a Lagrangian density of the form (6), if one adopts a two-component representation of the state function as proposed in H. Feshbach and F. Villars, Rev. Mod. Phys. 30, 24 (1958).

[43] O. Klein, Elektrodynamik und Wellenmechanik vom Standpunkt des Korrespondenzprinzips, Z. Phys. 41, 407 (1927).

[44] W. Gordon, Der Comptoneffekt nach der Schrödingerschen Theorie, Z. Phys. 40, 117 (1926).

[45] H. Goldstein, Classical Mechanics (Addison-Wesley, Reading, MA, 1950).

[46] F. H. Gaioli and E. T. G. Alvarez, Classical and quantum theories of spin, Found. Phys. 28, 1539 (1998).

[47] G. Manfredi and F. Haas, Self-consistent fluid model for a quantum electron gas, Phys. Rev. B 64, 075316 (2001). 